\title{
Inclusive diffraction in DIS - H1 Results
}

\author{
Paul Laycock \\ University of Liverpool \\ Oliver Lodge Laboratory, Department of Physics, \\ Oxford St. Liverpool L69 7ZE, United Kingdom \\ E-mail:laycock@mail.desy.de
}

Results are presented of three analyses on the diffractive deep-inelastic scattering cross section measured by the $\mathrm{H} 1$ experiment at HERA. In the first analysis [1], the process ep $\rightarrow e X Y$ is studied where $Y$ is a proton or low mass proton excitation carrying a fraction $1-x_{I P}>0.95$ of the incident proton longitudinal momentum and the squared four-momentum transfer at the proton vertex satisfies $|t|<1 \mathrm{GeV}^{2}$. Events are selected based on the presence of a large rapidity gap between the two hadronic final state systems $X$ and $Y$. The cross section is measured for photon virtualities in the range $3.5 \leq Q^{2} \leq 1600 \mathrm{GeV}^{2}$, triple differentially in $x_{I P}, Q^{2}$ and $\beta=x / x_{I P}$, where $x$ is the Bjorken scaling variable. At low $x_{I P}$, the data are consistent with a factorisable $x_{I P}$ dependence. In the second analysis [2], the process ep $\rightarrow e X p$ is measured by selecting events with a leading final state proton detected in the H1 Forward Proton Spectrometer. The cross section is measured also as a function of $t$; the $\beta$ and $Q^{2}$ dependences factorise from the $t$ dependence within uncertainties. The ratio of the cross sections measured by these two analyses, and thus the $M_{Y}$ dependence of the diffractive cross section, is seen to be independent of $x_{I P,} \beta$ and $Q^{2}$ dependences within uncertainties. Diffractive parton distribution functions and their uncertainties are determined from a next-to-leading order DGLAP QCD analysis of the $Q^{2}$ and $\beta$ dependences of the cross section measured in the first analysis. The resulting gluon distribution carries an integrated fraction of around $70 \%$ of the exchanged momentum in the $Q^{2}$ range measured. Total and differential cross sections are also measured for the diffractive charged current process $e p \rightarrow v X Y$ and are found to be well described by predictions based on the diffractive parton distributions. The ratio of the diffractive to the inclusive neutral current $e p$ cross sections is studied. Over most of the kinematic range, this ratio shows no significant dependence on $Q^{2}$ at fixed $x_{I P}$ and $x$ or on $x$ at fixed $Q^{2}$ and $\beta$. Finally, results of a preliminary $\mathrm{H} 1$ analysis [3] of the process $e p \rightarrow e X Y$ using both the large rapidity gap selection method and a method based on decomposing the $\ln M_{x}^{2}$ distribution are shown. There is reasonable agreement between the two measurements in the phase space in which both experimental techniques can be reliably employed; agreement is also observed with measurements made by the ZEUS collaboration using this ' $M_{X}$ ' technique [4].

International Workshop on Diffraction in High-Energy Physics-Diffraction 06-

Adamantas, Milos island, Greece

5-10 September 2006 


\section{Introduction}

A complete description of the analyses described here can be found in the two recent $\mathrm{H} 1$ publications on inclusive diffractive DIS cross sections [1,2].

Figure 1 shows a schematic of the neutral current diffractive DIS process in two factorization schemes. The first scheme shown in Figure 1 (a) follows a proof [5] of a hard scattering QCD collinear factorisation theorem [6,7,8] for semi-inclusive DIS cross sections such as that for $e p \rightarrow e X p$. This theorem implies that the concept of 'diffractive parton distribution functions' (DPDFs) may be introduced. Further to this proof a factorisation has been found to hold to good approximation, namely the 'proton vertex factorisation' sketched in Figure 1 (b). This factorisation implies that the $\beta$ and $Q^{2}$ dependences of the diffractive cross section factorise from the $x_{I P}, t$ and $M_{Y}$ dependences. The analyses published in [1,2] validate the proton vertex factorisation approximation, thus allowing for DPDFs to be extracted in [1].

(a)

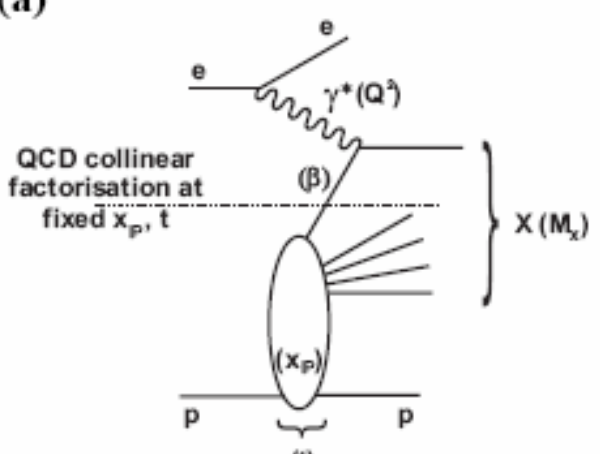

(b)

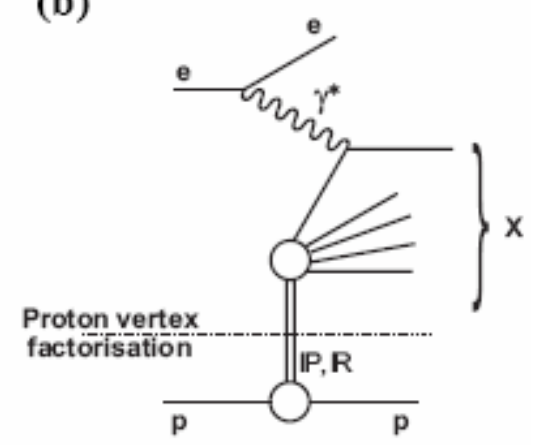

Figure 1. Schematic illustration of the neutral current diffractive DIS process ep $\rightarrow e X Y$, proceeding via virtual photon exchange. The dotted lines in (a) and (b) show the points at which the diagram can be divided under the assumptions of $Q C D$ hard scattering collinear factorisation and proton vertex factorisation, respectively. The kinematic variables defined in section 1.1 are also indicated in (a).

\subsection{Kinematics and cross section definitions}

The usual DIS kinematic variables are defined as

$$
Q^{2}=-q^{2}, \quad x=\frac{-q^{2}}{2 P \cdot q}, \quad y=\frac{P \cdot q}{P \cdot k},
$$

where $Q^{2}$ is the boson virtuality, $x$ is the longitudinal momentum fraction of the proton carried by the struck quark and $y$ measures the inelasticity of the process. The squared invariant masses of the positron-proton and gauge boson-proton systems are $s=(k+P)^{2}$ and $W^{2}=(q+P)^{2}$, respectively. The kinematics peculiar to diffraction, namely the longitudinal momentum fractions of the diffractive exchange with respect to the proton, $x_{I P}$, and of the struck quark with respect to the diffractive exchange, $\beta$, are defined as

$$
x_{I P}=\frac{q \cdot\left(P-p_{Y}\right)}{q \cdot P}, \quad \quad \beta=\frac{Q^{2}}{2 q \cdot\left(P-p_{Y}\right)},
$$


where $p_{Y}$ is the four-momoentum of the $Y$ system and $\beta x_{I P}=x$. Finally, the four-momentum transfer at the proton vertex, $t$, is defined as $t=\left(P-p_{Y}\right)^{2}$.

The neutral current diffractive DIS cross section can be written in terms of these kinematic variables:

$$
\frac{d^{3} \sigma^{e p \rightarrow e X Y}}{d x_{I P} d x d Q^{2}}=\frac{2 \pi \alpha^{2}}{x Q^{4}} \cdot Y_{+} \cdot \sigma_{r}^{D(3)}\left(x_{I P}, x, Q^{2}\right),
$$

where $Y_{+}=1+(1-y)^{2}$. The reduced cross section, $\sigma_{r}^{D(3)}$, can be related to the structure functions in the one photon exchange approximation according to

$$
\sigma_{r}^{D(3)}=F_{2}^{D(3)}-\frac{y^{2}}{Y_{+}} F_{L}^{D(3)} \text {. }
$$

For $y$ not too close to unity, ${\sigma_{r}}^{D(3)}=F_{2}{ }^{D(3)}$ holds to a very good approximation.

\section{Factorisation of the diffractive DIS cross section}

The events used in analysis 1 to measure the $e p \rightarrow e X Y$ cross section, although dominated by the elastic proton process (i.e. $Y=$ proton), by nature of the selection method contain some proton dissociation contributions. The cross section is defined for:

$$
M_{Y}<1.6 \mathrm{GeV}, \quad|t|<1 \mathrm{GeV}^{2} .
$$

Analyses 1 and 2 are in good agreement on the $t$-integrated cross section, after accounting for proton dissociation contributions in the first analysis, and are in good agreement with an analysis using tagged protons published by the ZEUS collaboration [9].

\subsection{Factorisation of the $M_{Y}$ dependence}

Taking the ratio of the cross section for this process and the process $e p \rightarrow e X p$ allows a simple experimental test of the factorisation of the $M_{Y}$ dependence of the diffractive DIS cross section. Figure 2 shows that the $M_{Y}$ dependence factorises from the $Q^{2}$ and $\beta$ dependences within experimental uncertainties (the $x_{I P}$ dependence is also seen to factorise, figure not shown).
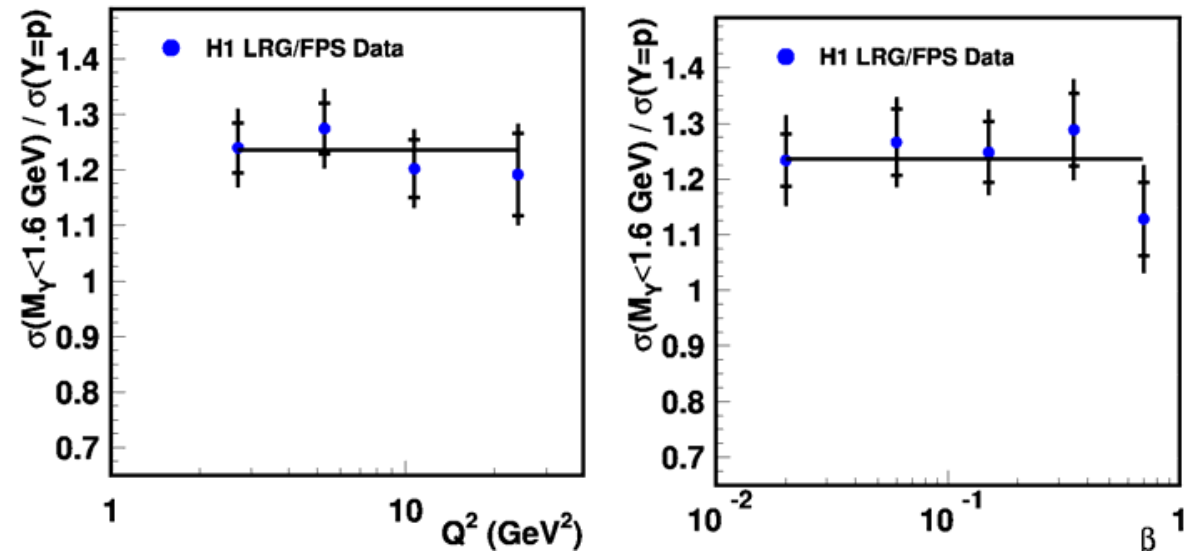

Figure 2. The ratio of the diffractive cross section for $M_{Y}<1.6 \mathrm{GeV}$ and $|t|<1 \mathrm{GeV}^{2}$ to that for $Y=p$ and $|t|<1 \mathrm{GeV}^{2}$. The $M_{Y}$ dependence of the diffractive DIS cross section factorises from the $Q^{2}, \beta$ and $x_{I P}$ dependences within uncertainties. 


\section{$2.2 \quad$ Factorisation of the $t$-dependence}

Figure 3 shows the $t$-dependence of the data in analysis 2 , parameterised as $\mathrm{d} \sigma / \mathrm{d} t \sim e^{-B t}$, as a function of $Q^{2}$ and $\beta$ at fixed $x_{I P}$. Within uncertainties there is no dependence of the $B$ parameter on either $Q^{2}$ or $\beta$ at fixed $x_{I P}$ observed.
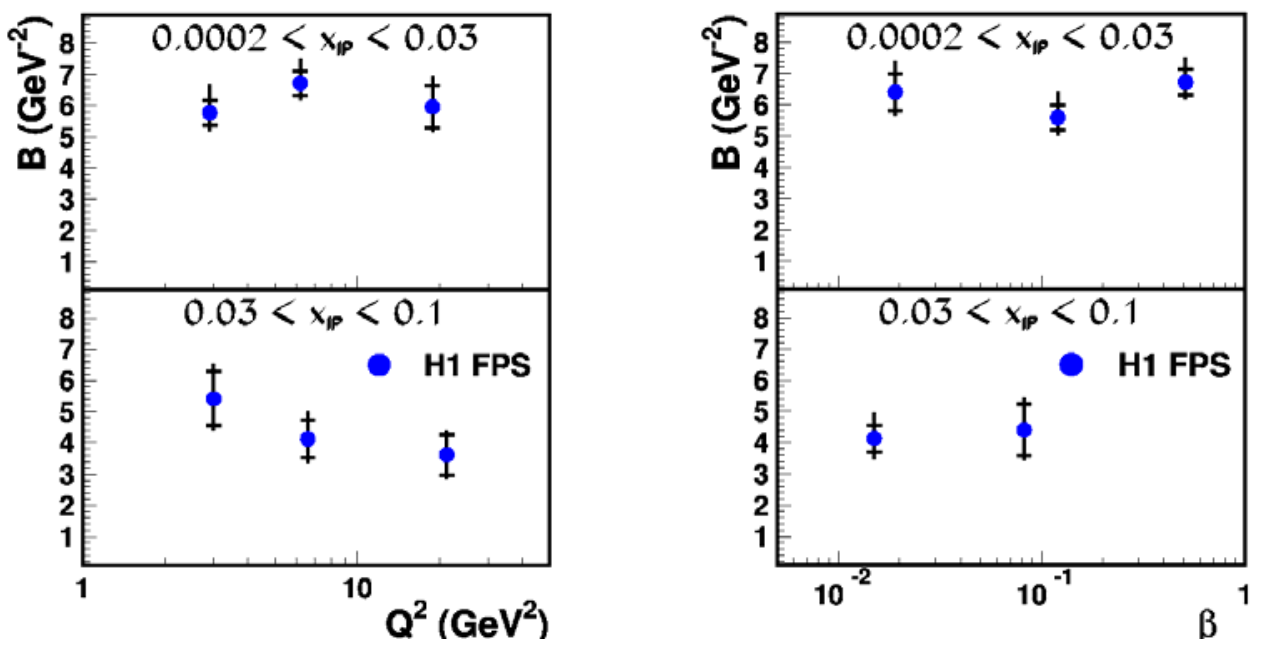

Figure 3. The $t$ dependence of the data as a function of $Q^{2}$ and $\beta$ at fixed $x_{I P}$. The $t$ dependence of the diffractive DIS cross section factorises from the $Q^{2}$ and $\beta$ dependences at fixed $x_{I P}$ within uncertainties.

\subsection{Factorisation of the $x_{I P}$ dependence}

The $x_{I P}$ dependence of the data can be expressed in terms of an effective Pomeron intercept, $\alpha_{\mathrm{IP}}(0)$. Figure 4 shows that this is not dependent on $Q^{2}$ or $\beta$ within uncertainties.

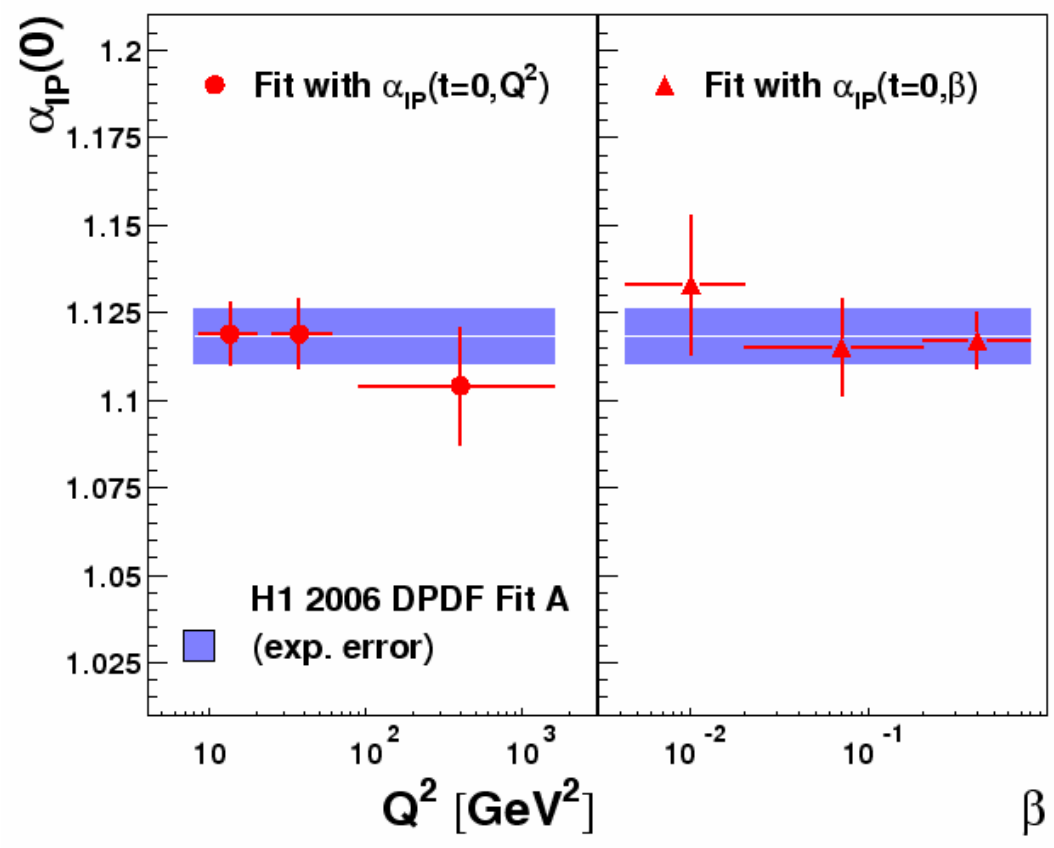

Figure 4. The $x_{I P}$ dependence of the data, $\alpha_{I P}(0)$, as a function of $Q^{2}$ and $\beta$. The $x_{I P}$ dependence of the data is seen to factorise from the $Q^{2}$ and $\beta$ dependences. 


\section{3. $\quad Q^{2}$ and $\beta$ dependences of the diffractive cross section}

Figure 5 shows the $Q^{2}$ and $\beta$ dependences of the diffractive cross section at fixed $x_{I P}$ for one value of $x_{I P}=0.003$. The data are shown compared to the fit described in [1], which uses the proton vertex factorisation approximation to allow a NLO QCD DGLAP fit to the $Q^{2}$ and $\beta$ dependences of the data. The fit quality is very good and can be seen to describe the data very well, except at the lowest $Q^{2}$ [1].
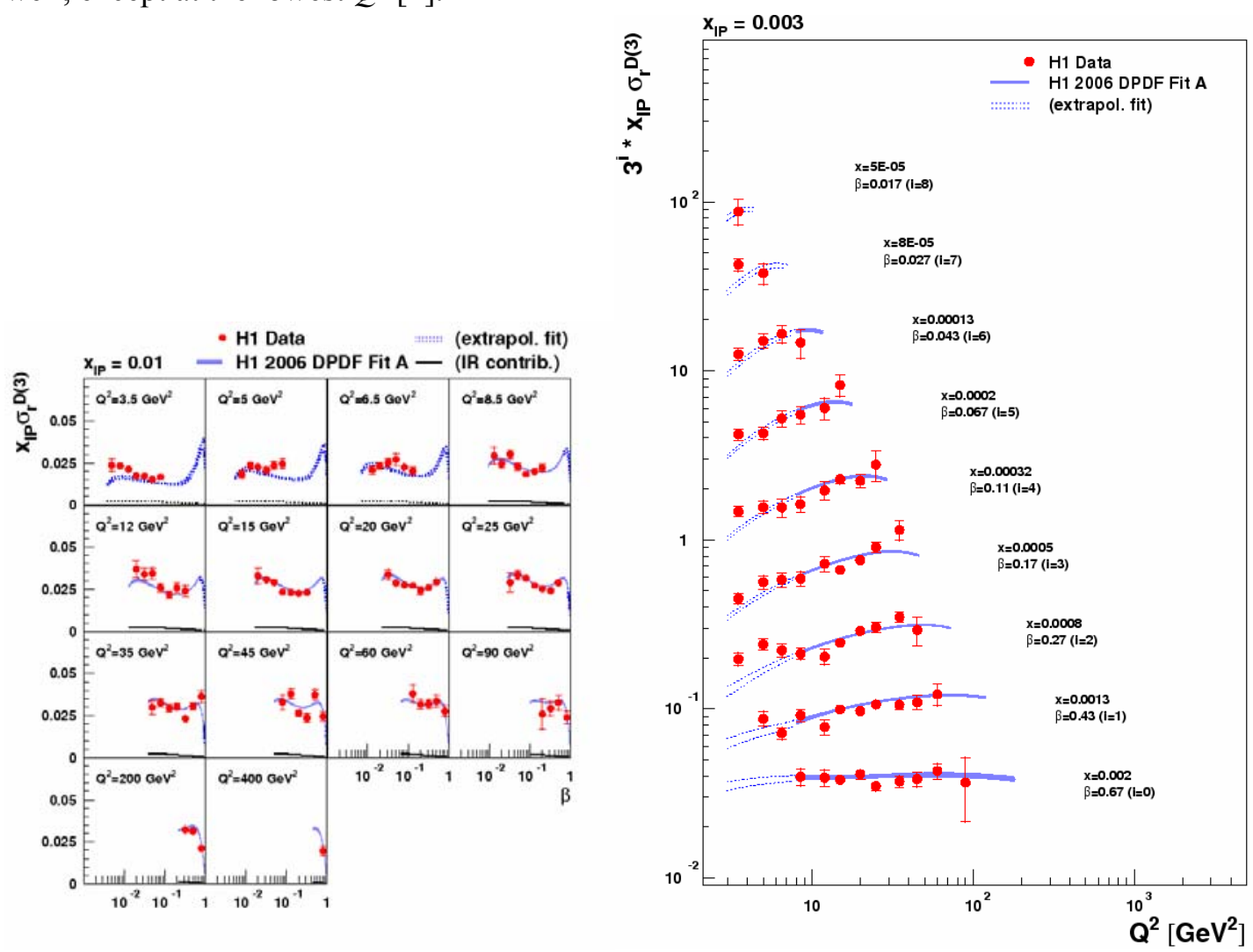

Figure 5. The $\beta$ (left) and $Q^{2}$ dependences of the diffractive cross section at fixed $x_{I P}$. The data are shown compared to a NLOQCD fit to the $Q^{2}$ and $\beta$ dependences of the data.

\subsection{The logarithmic $Q^{2}$ derivative}

Figure 6 shows the logarithmic $Q^{2}$ derivative of the diffractive DIS cross section as a function of $\beta$, divided by a term which encapsulates the $x_{I P}$ dependence of the data according to the proton vertex factorisation ansatz, at fixed $x_{I P}$. The left plot shows that large positive scaling violations are present in the data up to $\beta \sim 0.6$ and the size of these scaling violations are compatible at different values of $x_{I P}$, supporting the proton vertex factorisation approximation. The right plot shows the data compared to the predictions of the NLO QCD fit; contributions arising from gluons and quarks are shown separately. While the logarithmic $Q^{2}$ derivative is dominated by the gluon-driven contribution at low and medium $\beta$, at large $\beta>0.3$ this quantity is dominated by the quark-driven term. Thus, the sensitivity of this quantity to the gluon density at large $\beta$ is expected to be poor. 

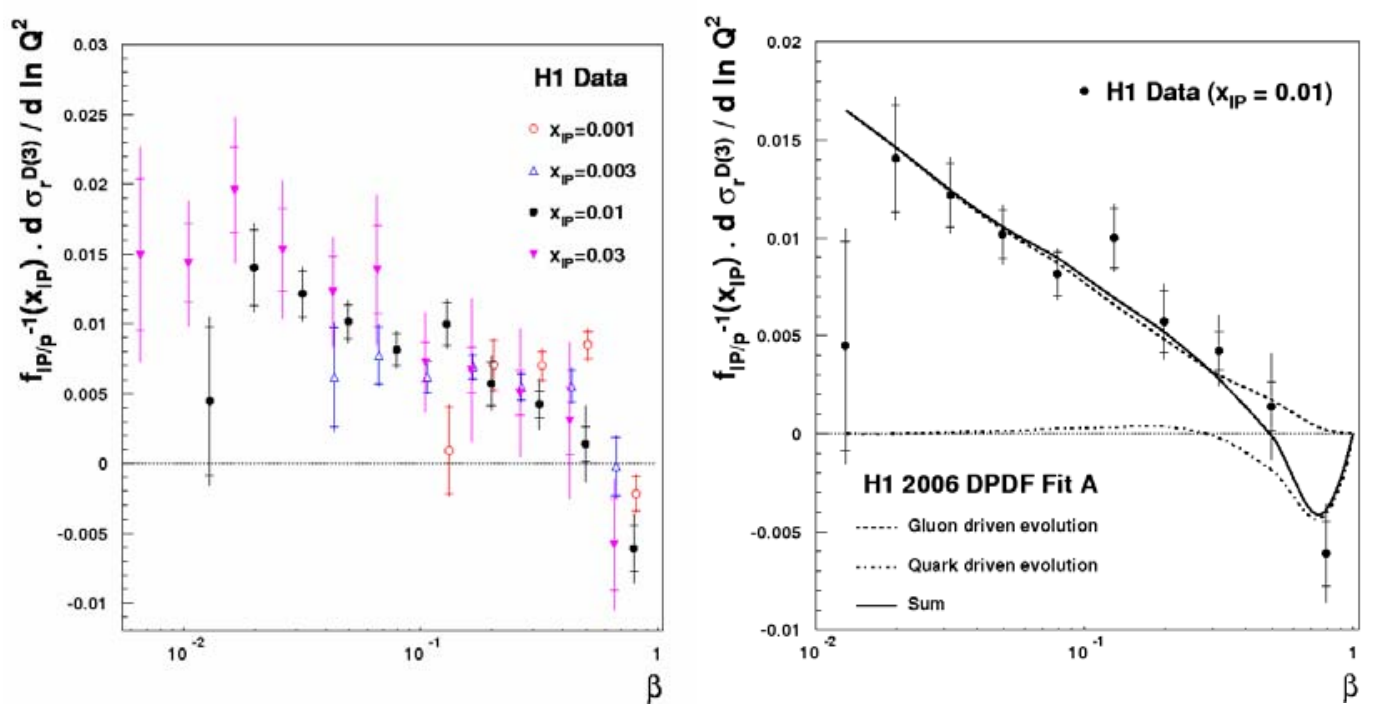

Figure 6. The logarithmic $Q^{2}$ derivative of the diffractive DIS cross section as a function of $\beta$, divided by a term which encapsulates the $x_{I P}$ dependence of the data, at fixed $x_{I P}$.

\subsection{Diffractive parton density functions}

The NLO QCD fit allows the extraction of the DPDFs shown in Figure 7. Two fits of similar quality, Fit A and Fit B, were produced in [1] and are seen to give very similar results on a well constrained quark singlet DPDF. The gluon DPDF is seen to be less well constrained, especially at high momentum fractions; this is expected given the insensitivity of the logarithmic $Q^{2}$ derivative to the gluon contribution at large momentum fractions, $z$.
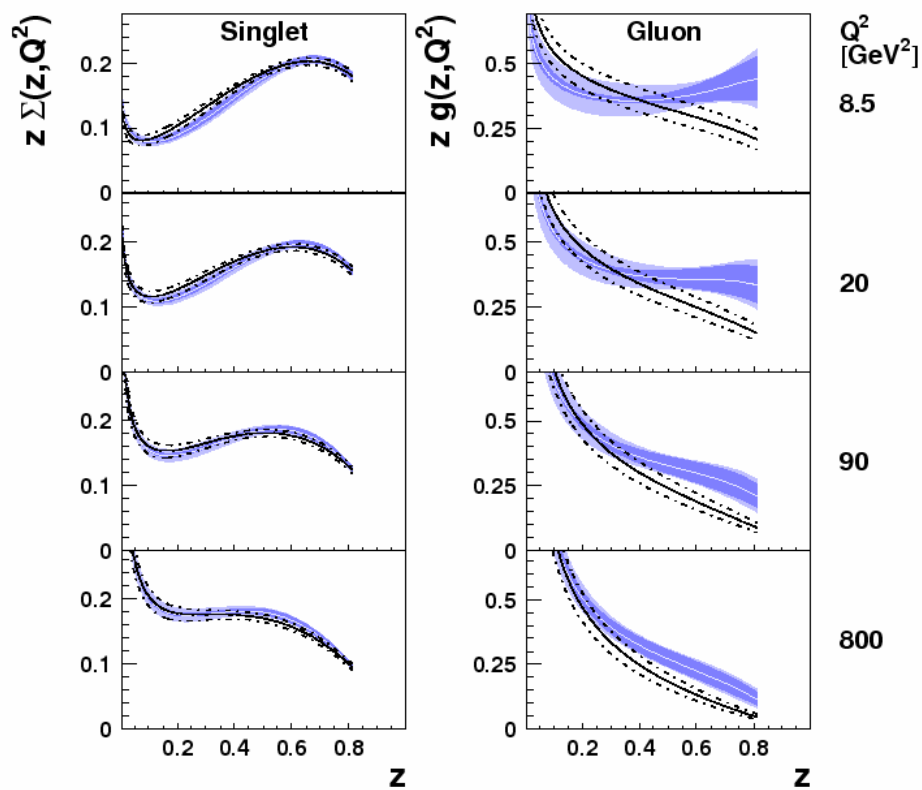

H1 2006 DPDF Fit A

exp. error)

H1 2006 DPDF Fit B

-... (exp.+theor. error)

Figure 7. The Diffractive parton density functions as a function of momentum fraction $z$. The DPDFs resulting from Fits $A$ and $B$ are shown; there is considerable uncertainty on the gluon at high $z$. 


\section{The ratio of diffractive to inclusive cross sections}

Figure 8 shows the logarithmic $Q^{2}$ derivative of the ratio of diffractive to inclusive cross sections as a function of $\beta$ at fixed $x_{I P}$; again the data have been divided by a term which encapsulates the $x_{I P}$ dependence according to the proton vertex factorisation ansatz. This quantity is consistent with zero over much of the kinematic range, implying that the ratio of quarks to gluons in the parton densities is the same in both the diffractive and inclusive cases. In the case of diffraction the ratio of quarks to gluons in the parton densities is measured to be approximately 30:70, which is compatible at low $x$ with global fits to inclusive DIS data [1].

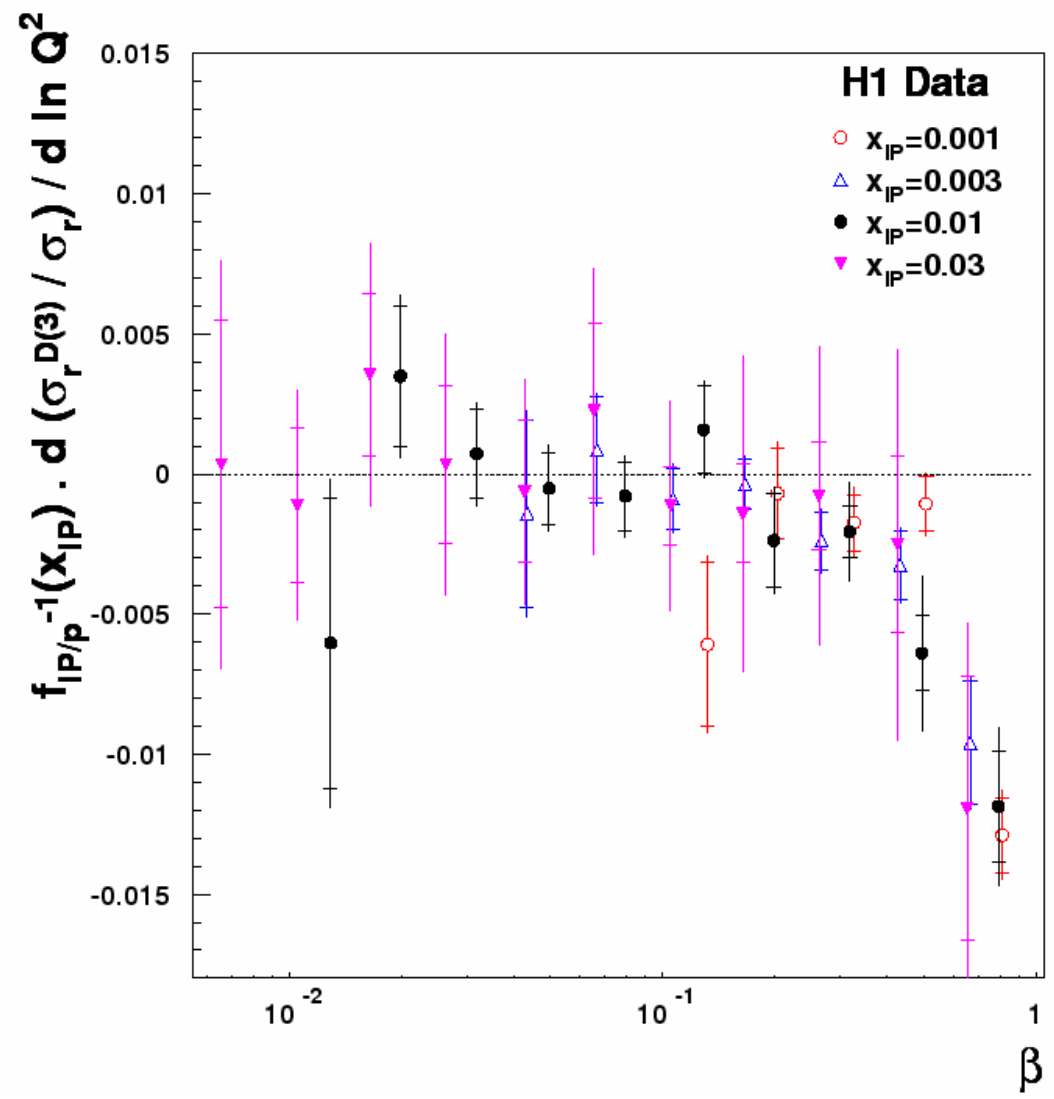

Figure 8. The logarithmic $Q^{2}$ dependence of the ratio of diffractive to inclusive cross sections as a function of $\beta$, divided by a term which encapsulates the $x_{I P}$ dependence of the data, at fixed $x_{I P}$. 


\section{The diffractive charged current cross section}

The diffractive charged current process $e p \rightarrow v X Y$ is shown in Figure 9. The diffractive charged current process is sensitive to the flavour composition of the quark singlet contribution to the diffractive exchange, which is completely unconstrained by the neutral current data. Thus, a measurement of the diffractive charged current process tests the quark singlet flavour composition assumed in [1]. The differential cross sections shown in Figure 9 as a function of $x_{I P}, Q^{2}$ and $\beta$ agree well with the prediction of the NLO QCD fit to neutral current data.

The prediction is obtained using the RAPGAP [10] Monte Carlo generator. The cross section prediction for the kinematic range $Q^{2}>200 \mathrm{GeV}^{2}, y<0.9$ and $x_{I P}<0.05$ at $\sqrt{\mathrm{s}}_{\mathrm{s}}=319$ $\mathrm{GeV}$ is $500 \mathrm{fb}$, which is compatible with the measured value of

$$
\sigma_{C C}^{\text {diff }}=390 \pm 120 \text { (stat.) } \pm 70 \text { (sys.) fb, }
$$

corresponding to $2.2 \pm 0.7$ (stat.) \pm 0.4 (sys.) $\%$ of the total charged current cross section for the same $Q^{2}$ and $y$ ranges and $x<0.05$.
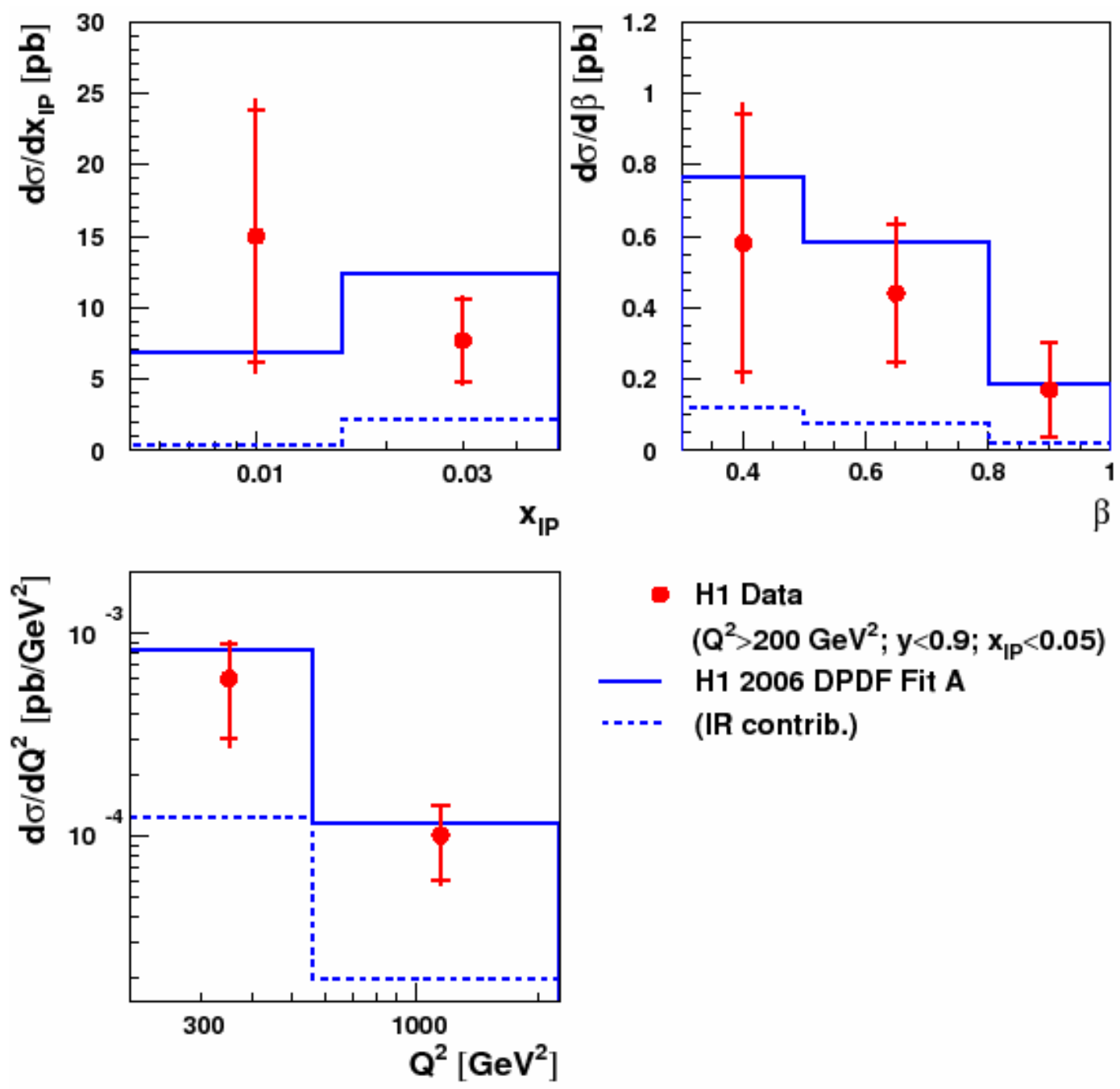

- H1 Data $\left(Q^{2}>200 \mathrm{GeV}^{2} ; \mathrm{y}<0.9 ; \mathrm{x}_{\mathrm{IP}}<0.05\right)$

- H1 2006 DPDF Fit A

(IR contrib.)

Figure 9. The diffractive charged current cross section shown differentially in $x_{I P}, \beta$ and $Q^{2}$. Shown as a solid histogram is the prediction of a QCD fit to neutral current data; the dashed histogram shows the contribution from the sub-leading (IR) component. 


\subsection{New preliminary results comparing the LRG and $M_{X}$ methods}

There are two commonly-used experimental techniques used to select diffractive DIS events with high acceptance. The technique used by $\mathrm{H} 1$ in analysis 1 exploits a topological characteristic of diffractive events, namely the fact that there is a large rapidity gap (LRG) separating the two hadronic final state systems $X$ and $Y$. A second technique, the ' $\mathrm{M}_{\mathrm{X}}$ ' method used by the ZEUS collaboration to select diffractive DIS events, decomposes the $\ln M_{X}^{2}$ distribution into diffractive and inclusive contributions [4]. Comparisons of these two high acceptance methods with analyses using a tagged leading final state proton suggest that there are differences. In particular, while the LRG and tagged proton methods agree well (up to a global normalisation correction factor to account for a small proton dissociation contribution in the LRG sample) systematic differences between the cross sections measured using the $M_{X}$ method and the other two methods are apparent [11].

A new preliminary $\mathrm{H} 1$ analysis uses both the LRG and $M_{X}$ experimental techniques to analyse one data set [3]. The H1 $M_{X}$ analysis uses a more restricted phase space than the ZEUS analysis due to differences in the detector apparatus (namely the lack of precision calorimetry in the very forward region). Figure 10 shows the results of the two measured cross sections compared with one another and a publication by the ZEUS collaboration using the $M_{X}$ method [9]. There is reasonable agreement between all three measurements in the limited phase space where comparisons can be made.

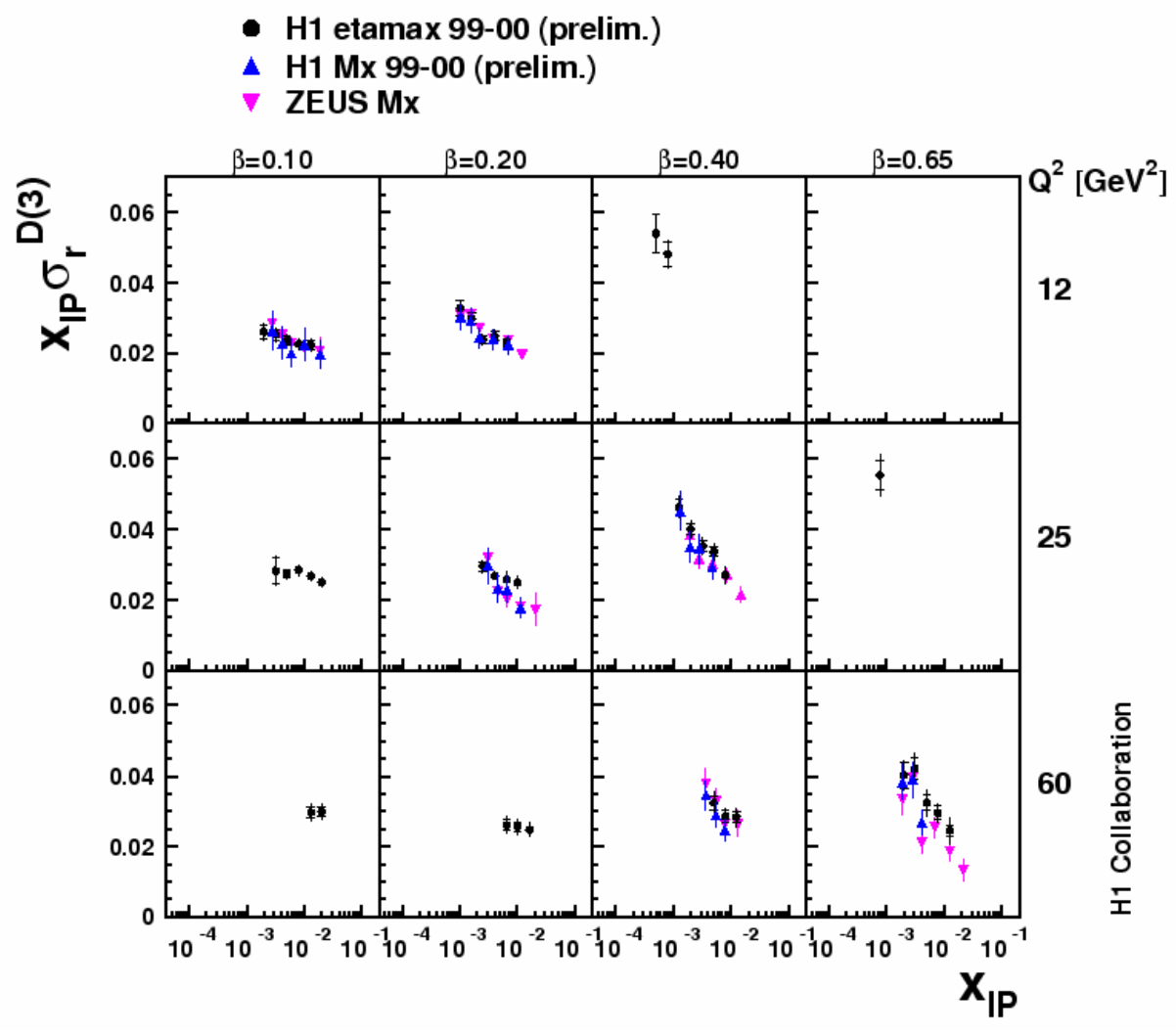

Figure 10. A comparison of the $L R G$ (etamax) selection method with an analysis using the $M_{X}$ method on the same data from $\mathrm{HI}$, and a publication using the $M_{X}$ method by the ZEUS collaboration. 


\section{Summary}

The cross section for the process $e p \rightarrow e X Y$ has been measured under the conditions $M_{Y}<1.6$ $\mathrm{GeV}$ and $|t|<1 \mathrm{GeV}^{2}[1]$ and agrees well with a measurement [2] of the process $e p \rightarrow e X p$ after correcting for the small proton dissociation contributions in the former measurement. The diffractive DIS cross section is seen to have $Q^{2}$ and $\beta$ dependences which are independent within uncertainties of $M_{Y}, t$ and $x_{I P}$, supporting the proton vertex factorisation approximation. NLO QCD fits employing this factorisation give a good description of the data and predict the results of the diffractive charged current process $e p \rightarrow v X Y$ well. DPDFs extracted from two such NLO QCD fits show that the singlet quark distribution is well constrained by measurements of inclusive diffractive DIS, while the gluon is less well constrained, especially at large momentum fractions, $z$. This is a result of the logarithmic $Q^{2}$ derivative of the cross section which, while dominated by the gluon contribution at low and medium $z$, is dominated by the quark singlet contribution at high $z$. Further constraints on QCD fits from diffractive dijet data can remedy this problem (see contribution from Matthias Mozer, these proceedings).

The ratio of diffractive to inclusive cross sections was also studied. In particular, the logarithmic $Q^{2}$ derivative of this ratio at fixed $x_{I P}$ implies that the ratio of gluons to quarks in both the diffractive and inclusive cases is the same $(\sim 70: 30)$. Finally, a preliminary analysis using both the large rapidity gap and $M_{X}$ techniques on the same data set shows good agreement between the two experimental techniques in the limited region where comparisons are possible.

\section{References}

[1] H1 Collaboration, Measurement and QCD Analysis of the Diffractive Deep-Inelastic Scattering Cross Section at HERA, DESY 06-049, submitted to Eur. Phys. J. C. [hep-ex/ 0606004 ].

[2] H1 Collaboration, Diffractive Deep-Inelastic Scattering with a Leading Proton at HERA, DESY 06048, submitted to Eur. Phys. J. C. [hep-ex/ 0606003 ].

[3] E. Sauvan, Measurement of Inclusive Diffractive Deep-Inelastic Scattering at HERA (99-04 data), presented at DIS 2006, Spring 2006.

[4] S. Chekanov et al. [ZEUS Collaboration], Eur. Phys. J. C. 38 (2004) 43 [hep-ex/ 0501060 ].

[5] J. Collins, Phys. Rev. D 57 (1998) 3051 [Erratum-ibid. D 61 (2000) 019902] [hep-ph/9709499].

[6] A. Berera and D. Soper, Phys. Rev. D 50 (1994) 4328 [hep-ph/ 9403276$].$

[7] A. Berera and D. Soper, Phys. Rev. D 53 (1996) 6162 [hep-ph/9509239].

[8] Z. Kunszt and W. J. Stirling, Proc. Of the Workshop on Deep-Inelastic Scattering and Related Phenomenoa DIS96, eds. G. D’Agostini, A. Nigro, Rome (1996) 240 [hep-ph/ 9609245 ].

[9] S. Chekanov et al. [ZEUS Collaboration], Eur. Phys. J. C. 38 (2004) 43 [hep-ex/ 0408009 ].

[10] H. Jung, Comput. Phys. Commun. 86 (1995) 147; (see also http://www.desy.de/ jung/rapgap.html).

[11] P. Newman and F. P. Schilling, Proc. Of the HERA-LHC workshop, eds. A. De Roeck, H. Jung, CERN-2005-014 (2005) 482 [hep-ex/0511032]. 